\title{
Seletividade de oito inseticidas a predadores de lagartas em $\operatorname{citros}^{(1)}$
}

\author{
Tederson Luiz Galvan ${ }^{(2)}$, Marcelo Coutinho Picanço(3), Leandro Bacci(3), \\ Eliseu José Guedes Pereira( ${ }^{(3)}$ e André Luiz Barreto Crespo ${ }^{(3)}$
}

Resumo - O objetivo deste trabalho foi avaliar a seletividade dos inseticidas abamectina, acefato, carbaril, deltametrina, fenitrotiom, fenpropatrina, paratiom metílico e triclorfom aos predadores Brachygastra lecheguana (Latreille), Protonectarina sylveirae (Saussure) e Protopolybia exigua (Saussure) (Hymenoptera: Vespidae) em concentrações que corresponderam a $50 \%$ e $100 \%$ da dose recomendada para o controle de lagartas em citros. Os inseticidas estudados não foram seletivos em favor do predador P. sylveirae. Fenitrotiom, fenpropatrina, paratiom metílico e triclorfom não foram seletivos em favor dos predadores B. lecheguana e $P$. exigua. Abamectina, acefato e carbaril foram medianamente seletivos em favor de P. exigua, o mesmo foi verificado com abamectina e carbaril em favor de B. lecheguana. Acefato foi seletivo em favor de B. lecheguana e deltametrina em favor de P. exigua. As altas mortalidades causadas por deltametrina a $P$. sylveirae, triclorfom a $B$. lecheguana e acefato a $P$. exigua decresceram quando aplicados em subdose. B. lecheguana foi o predador mais tolerante à dose de acefato, seguido por $P$. exigua e $P$. sylveirae. O predador $P$. exigua foi mais tolerante à dose de deltametrina que $B$. lecheguana e $P$. sylveirae. As três espécies de predadores foram altamente suscetíveis às doses e subdoses de fenitrotiom, fenpropatrina e paratiom metílico, e à dose de triclorfom.

Termos para indexação: Brachygastra lecheguana, Protonectarina sylveirae, Protopolybia exigua, controle de insetos, controle biológico.

\section{Selectivity of eight insecticides to predators of citrus caterpillars}

\begin{abstract}
The selectivity of abamectin, acephate, carbaryl, deltamethrin, fenitrothion, fenpropathrin, methyl parathion and trichlorfon to the predators Brachygastra lecheguana (Latreille), Protonectarina sylveirae (Saussure) and Protopolybia exigua (Saussure) (Hymenoptera: Vespidae) was evaluated using insecticide concentrations corresponding to $50 \%$ and $100 \%$ of the current recommended rate for citrus caterpillars. The insecticides studied were not selective in favor of the predator $P$. sylveirae. Fenitrothion, fenpropathrin, methyl parathion and trichlorfon were not selective in favor of the predator $B$. lecheguana and $P$. exigua. Abamectin, acephate and carbaryl showed median selectivity in favor of $P$. exigua, and the same was observed with abamectin and carbaryl in favor of $B$. lecheguana. Acephate was selective in favor of B. lecheguana and deltamethrin in favor of P. exigua. The high mortalities caused by deltamethrin in $P$. sylveirae, trichlorfon in B. lecheguana and acephate in $P$. exigua decreased when the lower concentration was used. B. lecheguana was the most tolerant predator to the higher acephate concentration, followed by P. exigua and P. sylveirae. P. exigua was more tolerant to the higher deltamethrin concentration than B. lecheguana and $P$. sylveirae. The three predator species were highly susceptible to both concentrations of fenitrothion, fenpropathrin and methyl parathion, and the higher concentration of trichlorfon.
\end{abstract}

Index terms: Brachygastra lecheguana, Protonectarina sylveirae, Protopolybia exigua, insect control, biological control.

\footnotetext{
(1) Aceito para publicação em 2 de maio de 2001.

(2) Universidade Federal de Viçosa (UFV), Dep. de Biologia Animal, CEP 36571-000 Viçosa, MG. Bolsista da Fapemig. E-mail: e34649@alunos.ufv.br

(3) UFV, Dep. de Biologia Animal. Bolsista do CNPq E-mail: picanco@mail.ufv.br, leandrobacci@bol.com.br, e34637@alunos.ufv.br, abcrespo@hotmail.com
}

\section{Introdução}

Entre as lagartas (Lepidoptera) que atacam citros, destacam-se o bicho-minador-das-folhas, Phyllocnistis citrella Stainton (Gracillaridae), e o bicho-furão, Ecdytolopha aurantiana Lima (Olethreutidae). P. citrella confecciona galerias 
serpenteadas no limbo foliar, as quais servem de porta de entrada para infecções pela bactéria causadora do cancro cítrico, Xanthomonas axonopodis pv. citri (Rodrigues et al., 1998). Já a E. aurantiana broqueia frutos verdes e maduros, podendo atingir a polpa. Além dessas, os desfolhadores Papilio thoas brasiliensis Roth e Jordan (Papilionidae) e Phobetron hipparchia (Cr.) (Limacodidae) e o broqueador de frutos Eulia dimorpha Clarke (Tortricidae) são lagartas-praga de citros (Gallo et al., 1988).

O principal método de controle desses insetospraga é o químico, sendo abamectina, acefato, carbaril, deltametrina, fenitrotiom, fenpropatrina, paratiom metílico e triclorfom os principais inseticidas usados no controle dessas lagartas em citros (Andrei, 1999). No controle biológico natural das lagartas dos citros destacam-se, entre os predadores, os himenópteros Vespidae (Lolato \& Moraes, 1997). Entretanto, o uso inadequado de inseticidas em citros reduz as populações desses inimigos naturais ocasionando a ressurgência de pragas ou a erupção de pragas secundárias (Pedigo, 1999). Nesse sentido Reis et al. (1998) verificaram que o uso de produtos não seletivos em citros foi grandemente prejudicial ao ácaro-predador, Iphiseiodes zuluagai Denmark \& Muma (Acari: Phytoseiidae).

Assim, para proteção dos inimigos naturais fazse necessário o uso de inseticidas eficientes contra as espécies-praga e seletivos em favor de seus inimigos naturais. A seletividade pode ser classificada em seletividade ecológica e fisiológica. A primeira, relaciona-se a formas de utilização dos inseticidas de modo a reduzir a exposição do inimigo natural ao inseticida. A segunda, diz respeito ao uso de inseticidas que sejam mais tóxicos à praga do que a seus inimigos naturais (Pedigo, 1999).

No estudo de seletividade de inseticidas, o emprego das doses recomendadas para o controle das pragas permite avaliação do impacto desses produtos aos inimigos naturais no momento de sua aplicação. Já o uso de subdoses, por exemplo 50\% da dose recomendada para o controle da praga, possibilita a avaliação do impacto dos inseticidas quando metade de suas concentrações originais estiverem decompostas (Suinaga et al., 1996). Além disso, doses subletais do inseticida podem também ser prejudiciais aos inimigos naturais por reduzirem sua eficiência de con- trole e suas populações, em virtude dos efeitos deletérios sobre a reprodução e desenvolvimento destes (Elzen, 1989).

O objetivo deste trabalho foi avaliar a seletividade de inseticidas aos predadores Protonectarina sylveirae (Saussure), Brachygastra lecheguana (Latreille) e Protopolybia exigua (Saussure) (Hymenoptera: Vespidae) em concentrações que correspondem a $50 \%$ (subdose) e $100 \%$ (dose) da recomendação para o controle de lagartas em citros.

\section{Material e Métodos}

Esta pesquisa foi conduzida no laboratório de Manejo Integrado de Pragas da Universidade Federal de Viçosa (UFV), em Viçosa, MG, de outubro de 1999 a abril de 2000. O delineamento experimental foi inteiramente casualizado, com quatro repetições, em arranjo fatorial $8 \times 3 \times 2$ (inseticidas $\times$ espécies $\times$ doses dos inseticidas), além da testemunha. Foram utilizados adultos das vespas predadoras $P$. sylveirae, B. lecheguana e $P$. exigua capturados em ninhos no campus da UFV.

Os inseticidas foram empregados em concentrações que correspondem a $50 \%$ e $100 \%$ da dose recomendada para o controle de lagartas em citros. Os inseticidas estudados e suas concentrações em $\mathrm{mg}$ de ingrediente ativo/mL de calda foram: abamectina $18 \mathrm{CE}(0,0041$ e 0,0021$)$, acefato 750 PS $(0,375$ e 0,1875$)$, carbaril 850 PM $(1,2$ e 0,6$)$, deltametrina $25 \mathrm{CE}(0,0075$ e 0,0038$)$, fenitrotiom $500 \mathrm{CE}$ $(0,75$ e 0,375$)$, fenpropatrina $300 \mathrm{CE}(1,5$ e 0,75$)$, paratiom metílico $600 \mathrm{CE}(0,6$ e 0,3$)$ e triclorfom $500 \mathrm{CE}(1,5 \mathrm{e}$ $0,75)$. Utilizou-se o espalhante adesivo, $\mathrm{N}$-dodecil benzeno sulfonato de sódio $320 \mathrm{CE}$, na concentração de $20 \mathrm{~mL} / 100 \mathrm{~L}$ de calda em todos os tratamentos (Andrei, 1999).

Folhas de laranja da variedade Pêra foram imersas em caldas inseticidas por cinco segundos, e na testemunha foram imersas em água mais espalhante adesivo. As folhas foram colocadas para secar por duas horas e, após a secagem, acondicionadas em placas de Petri $(9 \mathrm{~cm}$ de diâmetro por $2 \mathrm{~cm}$ de altura). Em cada placa foram liberados 10 insetos, constituindo-se assim a unidade experimental. As placas de Petri foram levadas para estufa incubadora a $25 \pm 0,5^{\circ} \mathrm{C}$ e umidade relativa de $75 \pm 5 \%$. Vinte e quatro horas após, foram feitas avaliações do número de insetos mortos por unidade experimental e os resultados foram corrigidos em relação a mortalidade ocorrida na testemunha, usando-se a fórmula de Abbott (1925).

Os resultados de mortalidade dos insetos foram transformados em arco seno $(\mathrm{x} / 100)^{0,5}$, para realização de análise de variância e comparação das médias pelo teste de Scott-Knott a $\mathrm{p}<0,05$ de significância. 


\section{Resultados e Discussão}

Verificou-se efeito significativo $(\mathrm{p}<0,05)$ na mortalidade dos Vespidae em razão de inseticidas, doses, interações entre inseticidas e espécies de predadores, inseticidas e doses, e doses, inseticidas e espécies.

Os inseticidas quando aplicados nas doses recomendadas para o controle de lagartas em citros não foram seletivos em favor de P. sylveirae $(100 \%$ de mortalidade). Fenitrotiom, fenpropatrina, paratiom metílico e triclorfom não foram seletivos em favor de B. lecheguana e P. exigua ( $100 \%$ de mortalidade). Abamectina, acefato e carbaril foram medianamente seletivos em favor de P. exigua $(58,92 \%$, $47,70 \%$ e $60,99 \%$ de mortalidade, respectivamente), o mesmo foi verificado com abamectina e carbaril em favor de B. lecheguana $(52,63 \%$ e $57,89 \%$ de mortalidade, respectivamente). Acefato foi seletivo em favor de B. lecheguana (5,85\% de mortalidade) e deltametrina em favor $P$. exigua $(1,55 \%$ de mortalidade) (Tabela 1).

Picanço et al. (1998) em relação a Polybia ignobilis (Haliday), Gonring et al. (1999) quanto a P. sylveirae, Polistes versicolor versicolor (Olivier) e P. exigua, e Moura et al. (2000) em relação a Polybia fastidiosuscula Saussure, Polybia scutellaris (White) e P. sylveirae observaram que deltametrina foi seletivo em favor desses Vespidae predadores.
Resultados semelhantes foram obtidos por Faleiro et al. (1995) com Doru luteipes (Scudder) (Dermaptera: Forficulidae); Suinaga et al. (1996), Picanço et al. (1996, 1997), Batalha et al. (1997) e Zanuncio et al. (1998) com pentatomídeos predadores.

A razão da seletividade de piretróides, como deltametrina, ainda não é conhecida. Entretanto, Yu (1988) relata que com Podisus maculiventris Say (Heteroptera: Pentatomidae) a metabolização dos piretróides por oxidases microssomais e esterases acarreta maior desintoxicação desses inseticidas nesse percevejo do que em suas presas. A insensibilidade do sistema nervoso central e a sensibilidade das enzimas (Na-K)-ATPase e Mg2-ATPase estão também relacionadas à ação neurotóxica de piretróides (Leng \& Xiao, 1995). A diferença na sensibilidade dos Vespidae predadores aos piretróides deltametrina e fenpropatrina, possivelmente, esteja relacionada às diferenças de seus pesos moleculares (505,2 e 349, respectivamente) (Farm..., 2001).

Diferentemente dos resultados obtidos neste trabalho, Hebling-Beraldo et al. (1981) não verificaram seletividade do acefato a Vespidae. Bayoun et al. (1995) sugerem que além da diferença na capacidade metabólica entre pragas e inimigos naturais, diferenças na solubilidade dos inseticidas em água podem estar relacionadas com sua seletividade. Assim, o acefato deve ter a menor taxa de penetração na cutícula lipídica dos insetos por possuir a maior

Tabela 1. Mortalidade média (\%) de três espécies de Hymenoptera: Vespidae causada pela dose recomendada e subdose ( $50 \%$ da dose) de oito inseticidas usados no controle de lagartas em citros. Viçosa, MG, 1999/2000(1).

\begin{tabular}{|c|c|c|c|c|c|c|}
\hline \multirow[t]{2}{*}{ Inseticida } & \multicolumn{2}{|c|}{ Brachygastra lecheguana } & \multicolumn{2}{|c|}{ Protonectarina sylveirae } & \multicolumn{2}{|c|}{ Protopolybia exigua } \\
\hline & Dose & Subdose & Dose & Subdose & Dose & Subdose \\
\hline Fenitrotiom $500 \mathrm{CE}$ & 100,00 a a & 97,37 a $\mathrm{aA}$ & $100,00 a \mathrm{aA}$ & $100,00 a \mathrm{aA}$ & $100,00 a \mathrm{aA}$ & $100,00 a \mathrm{aA}$ \\
\hline Paratiom metílico $600 \mathrm{CE}$ & $100,00 a \mathrm{aA}$ & $100,00 a \mathrm{aA}$ & $100,00 a \mathrm{aA}$ & $100,00 a \mathrm{aA}$ & $100,00 a \mathrm{aA}$ & $100,00 a \mathrm{aA}$ \\
\hline Fenpropatrina $300 \mathrm{CE}$ & $100,00 a \mathrm{aA}$ & $100,00 a \mathrm{aA}$ & $100,00 a \mathrm{aA}$ & $100,00 a \mathrm{aA}$ & $96,80 a \mathrm{aA}$ & $100,00 a \mathrm{aA}$ \\
\hline Triclorfom $500 \mathrm{CE}$ & $91,52 a \mathrm{aA}$ & $26,08 \mathrm{abB}$ & $100,00 a \mathrm{aA}$ & $95,67 a \mathrm{aA}$ & $100,00 a \mathrm{aA}$ & $100,00 a \mathrm{aA}$ \\
\hline Carbaril $850 \mathrm{PM}$ & $57,89 a \mathrm{bB}$ & $36,84 a b B$ & $100,00 a \mathrm{aA}$ & $100,00 a \mathrm{aA}$ & $60,99 \mathrm{abB}$ & $33,06 \mathrm{bbC}$ \\
\hline Abamectina $18 \mathrm{CE}$ & $52,63 a \mathrm{bB}$ & $42,11 \mathrm{abB}$ & $100,00 a \mathrm{aA}$ & $100,00 a \mathrm{aA}$ & $58,92 \mathrm{abB}$ & $55,39 a \mathrm{bB}$ \\
\hline Deltametrina $25 \mathrm{CE}$ & 100,00 a aA & $100,00 a \mathrm{aA}$ & $100,00 a \mathrm{aA}$ & $61,95 b \mathrm{bB}$ & $1,55 \mathrm{abC}$ & $5,32 a \mathrm{cD}$ \\
\hline Acefato $750 \mathrm{PS}$ & $5,85 a \mathrm{cC}$ & $7,89 a \mathrm{bC}$ & $100,00 a \mathrm{aA}$ & $100,00 a \mathrm{aA}$ & $47,70 \mathrm{abB}$ & $1,12 \mathrm{bbD}$ \\
\hline
\end{tabular}


solubilidade em água entre os inseticidas testados (65\% de solubilidade em água) (Farm..., 2001).

Como neste trabalho, com B. lecheguana e P. exigua, Leite et al. (1998) verificaram que a abamectina foi medianamente seletiva em favor do primeiro desses Vespidae. A seletividade da abamectina [mistura das avermectina Bla (80\%) e B1b (20\%)] pode ser decorrente do seu alto peso molecular $(873,1$ e 859,08$)$ e estrutura molecular complexa, que diminui a taxa de penetração na cutícula e a torna mais suscetível à ação de enzimas desintoxificativas, respectivamente (Hornsby et al., 1996).

Os resultados obtidos com os organofosforados fenitrotiom, paratiom metílico e triclorfom, que não foram seletivos em favor das vespas predadoras, concordam com os verificados por Gonring et al. (1999), que sugerem estar as altas toxicidades desses inseticidas aos predadores relacionadas com seus baixos pesos moleculares $(277,2,263,21$ e 257,44 , respectivamente) (Hornsby et al., 1996). Quanto menor o peso molecular, maior a taxa de penetração desses inseticidas na cutícula dos insetos.

Os resultados encontrados com o carbaril, que foi medianamente seletivo em favor de B. lecheguana e P. exigua, são semelhantes aos obtidos por Gonring et al. (1999) em relação às vespas predadoras de Grapholita molesta (Busch) (Lepidoptera: Olethreutidae), mas diferem dos obtidos por HeblingBeraldo et al. (1981) e Picanço et al. (1998) com vespas do gênero Polybia, para as quais o carbaril foi altamente tóxico.

As mortalidades causadas por fenitrotiom, fenpropatrina e paratiom metílico aos três predadores (triclorfom a P. exigua e $P$. sylveirae; abamectina, acefato e carbaril a $P$. sylveirae, e deltametrina a $B$. lecheguana) mantiveram-se elevadas mesmo quando se utilizou metade das doses (Tabela 1). Assim, infere-se que esses inseticidas apresentam alto impacto aos referidos predadores no momento da aplicação, o qual persiste mesmo após a decomposição de metade dos princípios ativos. As altas mortalidades causadas por deltametrina a $P$. sylveirae, triclorfom a $B$. lecheguana e acefato a $P$. exigua decresceram quando se utilizou a metade das doses (Tabela 1). Portanto, esses inseticidas apresentam alto impacto sobre tais predadores no momento da apli- cação, o qual torna-se reduzido com a decomposição de metade da concentração dos princípios ativos.

As mortalidades causadas pelas doses e subdoses de abamectina e carbaril a $B$. lecheguana e $P$. exigua foram semelhantes. Como esses inseticidas apresentaram-se medianamente seletivos a esses predadores (Tabela 1), infere-se que o impacto sobre essas espécies é mediano, tanto no momento da aplicação como quando ocorre decomposição de metade da concentração dos princípios ativos. O contrário ocorreu com o acefato em relação a $B$. lecheguana e deltametrina em relação a $P$. exigua, cujas mortalidades causadas pelas doses e subdoses foram semelhantes. Como esses inseticidas foram seletivos em favor desses predadores (Tabela 1), infere-se que o impacto sobre essas espécies é baixo no momento da aplicação, o qual não é reduzido com a decomposição de metade da concentração dos princípios ativos.

$B$. lecheguana e $P$. exigua foram mais tolerantes às doses e subdoses de abamectina e carbaril, e subdose de acefato do que $P$. sylveirae. B. lecheguana foi a espécie mais tolerante à dose de acefato enquanto $P$. sylveirae foi a mais suscetível, ficando $P$. exigua em situação intermediária. B. lecheguana foi mais tolerante à subdose de triclorfom do que $P$. exigua e $P$. sylveirae. P. exigua foi mais tolerante à dose de deltametrina do que B. lecheguana $\mathrm{e}$ P. sylveirae. P. exigua foi a espécie mais tolerante à subdose de deltametrina enquanto $P$. sylveirae foi a mais suscetível, ficando $B$. lecheguana em situação intermediária (Tabela 1). As três espécies de predadores foram altamente suscetíveis às doses e subdoses de fenitrotiom, fenpropatrina e paratiom metílico, e dose de triclorfom. Os mecanismos que explicam as diferenças de tolerância entre as espécies de vespas aos inseticidas são desconhecidos, entretanto devem ser semelhantes aos da seletividade de cada inseticida (Yu, 1988; Soderlund \& Bloomquist, 1990).

\section{Conclusões}

1. O acefato é seletivo em favor de Brachygastra lecheguana e medianamente seletivo em favor de Protopolybia exigua; deltametrina é seletivo em favor de P. exigua; abamectina e carbaril são medianamente seletivos em favor de B. lecheguana 
e P. exigua; abamectina, acefato, carbaril e deltametrina não são seletivos em favor de Protonectarina sylveirae; fenitrotiom, fenpropatrina, paratiom metílico e triclorfom são altamente tóxicos às três vespas predadoras.

2. As mortalidades causadas por fenitrotiom, fenpropatrina e paratiom metílico aos três predadores (triclorfom a $P$. exigua e $P$. sylveirae; abamectina, acefato e carbaril a $P$. sylveirae, e deltametrina a $B$. lecheguana) mantêm-se elevadas mesmo quando os inseticidas são aplicados em subdose.

3. Ocorre redução no impacto de deltametrina a $P$. sylveirae, triclorfom a $B$. lecheguana e acefato a $P$. exigua quando há decomposição da metade do princípio ativo.

4. B. lecheguana é a espécie mais tolerante ao acefato enquanto $P$. sylveirae é a mais suscetível, ficando $P$. exigua em situação intermediária. $P$. exigua é mais tolerante a deltametrina do que $B$. lecheguana e $P$. sylveirae. B. lecheguana e $P$. exigua são mais tolerantes a abamectina e carbaril do que $P$. sylveirae.

\section{Referências}

ABBOTT, W. S. A method of computing the effectiveness of an insecticide. Journal of Economic Entomology, Lanham, v. 18, n. 3 , p. $265-267,1925$.

ANDREI, E. Compêndio de defensivos agrícolas. 6. ed São Paulo: Andrei, 1999. 676 p.

BATALHA, V. C.; ZANUNCIO, J. C.; PICANÇO, M.; GUEDES, R. N. C. Selectivity of insecticides to Podisus nigrispinus (Heteroptera: Pentatomidae) and its prey Spodoptera frugiperda (Lepidoptera: Noctuidae). Ceiba, Tegucigalpa, v. 38, n. 1, p. 19-22, 1997.

BAYOUN, I. M.; PLAPP JUNIOR, F. W:; GILSTRAP, F. E.; MICHELS JUNIOR, G. J. Toxicity of selected insecticides to Diuraphis noxia (Homoptera: Aphididae) and its natural enemies. Journal of Economic Entomology, Lanham, v. 88, n. 5, p. 1177-1185, 1995.

ELZEN, G. W. Sublethal effects of pesticides on benefical parasitoids. In: JEPSON, P. C. (Ed.). Pesticides and nontarget invertebrates. Wimborne: Intercept, 1989. p. $129-150$

FALEIRO, F. G.; PICANÇO, M. C.; PAULA, S. V.; BATALHA, V. C. Seletividade de inseticidas a Spodoptera frugiperda (J. E. Smith) (Lepidoptera: Noctuidae) e o pre- dador Doru luteipes (Scudder) (Dermaptera: Forficulidae) Anais da Sociedade Entomológica do Brasil, Londrina, v. 24, n. 2 , p. $247-252,1995$

FARM chemicals handbook. Willoughby: Meister, 2001. $1000 \mathrm{p}$.

GALLO, D.; NAKANO, O.; SILVEIRA NETO, S.; CARVALHO, R. P. L.; BATISTA, C. G.; BERTI FILHO, E.; PARRA, J. R. P.; ZUCCHI, R. A.; ALVES, S. B.; VENDRAMIM, J. D. Manual de entomologia agrícola. 2. ed. São Paulo: Agronômica Ceres, 1988. 649 p

GONRING, A. H. R.; PICANÇO, M.; MOURA, M. F; BACCI, L.; BRUCKNER, C. H. Seletividade de inseticidas utilizados no controle de Grapholita molesta (Busch) (Lepidoptera: Olethreutidae) em pêssego: a Vespidae predadores. Anais da Sociedade Entomológica do Brasil, Londrina, v. 28, n. 2, p. 301-306, 1999.

HEBLING-BERALDO, M. J. A.; ROCHA, E. A.; MACHADO, V. L. L. Toxicidade de inseticidas (em laboratório) para Polybia (Myrapetra) paulista (Ihering, 1896) (Hymenoptera: Vespidae). Anais da Sociedade Entomológica do Brasil, Jaboticabal, v. 10, n. 2, p. 261-267, 1981

HORNSBY, A. G.; WAUCHOPE, R.; HERNER, A. E Pesticide properties in the environment. New York: Springer, 1996. $227 \mathrm{p}$

LEITE, G. L. D.; PICANCO, M.; GUEDES, R. N. C.; GUSMÃO, M. R. Selectivity of insecticides with and without mineral oil to Brachygastra lecheguana (Hymenoptera: Vespidae): a predator of Tuta absoluta (Lepidoptera: Gelechiidae). Ceiba, Tegucigalpa, v. 39, n. 1, p. 3-6, 1998

LENG, X. F.; XIAO, D. Q. Effect of deltamethrin on protein phosphorylation of housefly brain synaptosomes. Pesticide Science, Barking, v. 44, n. 1, p. 88-89, 1995

LOLATO, A.; MORAES, J. C. Ocorrência da "lagarta minadora das folhas de citros" Phyllocnistis citrella Stainton, 1856 (Lepidoptera: Gracilariidae) em Lavras, sul de Minas Gerais. Ciência e Agrotecnologia, Lavras, v. 21, n. 4, p. 521-522, 1997.

MOURA, M. F.; PICANÇO, M.; GONRING, A. H.; BRUCKNER, C. H. Seletividade de inseticidas a três Vespidae predadores de Dione juno juno (Lepidoptera: Heliconidae). Pesquisa Agropecuária Brasileira, Brasília, v. 35, n. 2 , p. $251-257,2000$

PEDIGO, L. P. Entomology and pest management. 3. ed. Englewood: Prentice Hall, 1999. 691 p. 
PICANÇO, M. C.; GUEDES, R. N. C.; BATALHA, V. C.; CAMPOS, R. P. Toxicity of insecticides to Dione juno juno (Lepidoptera: Heliconidae) and selectivity to two of its predaceous bugs. Tropical Science, Oxford, v. 36, n. 1, p. 51-53, 1996.

PICANÇO, M.; RIBEIRO, L. J.; LEITE, G. L. D.; GUSMÃO, M. R. Seletividade de inseticidas a Polybia ignobilis (Haliday) (Hymenoptera: Vespidae) predador de Ascia monuste orseis (Godart) (Lepidoptera: Pieridae). Anais da Sociedade Entomológica do Brasil, Londrina, v. 27, n. 1, p. 85-90, 1998.

PICANÇO, M.; RIBEIRO, L. J.; LEITE, G. L. D.; ZANUNCIO, J. C. Seletividade de inseticidas a Podisus nigrispinus predador de Ascia monuste orseis. Pesquisa Agropecuária Brasileira, Brasília, v. 32, n. 4, p. 369-372, abr. 1997.

REIS, P. R.; CHIAVEGATO, L. G.; MORAES, G. J.; ALVES, E. B.; SOUSA, E. O. Seletividade de agroquímicos ao ácaro predador Iphiseiodes zuluagai Denmark \& Muma (Acari: Phytoseiidae). Anais da Sociedade Entomológica do Brasil, Londrina, v. 27, n. 2, p. $265-274,1998$.

RODRIGUES, J. C. V.; ROSSETTI, V.; MACHADO, M. A.; TEÓFILO SOBRINHO, J.; NOGUEIRA, N. L.
Lagarta minadora dos citros: um fator do aumento de pragas e cancro cítrico. Laranja, Cordeirópolis, v. 19, n. 1, p. 49-60, 1998.

SODERLUND, D. M.; BLOOMQUIST, J. R. Molecular mechanisms of insecticide resistance. In: ROUSH, R. T.; TABASHNIK, B. E. (Ed.). Pesticide resistance in arthropods. New York: Chapman and Hall, 1990. p. 58-96.

SUINAGA, F. A.; PICANÇO, M.; ZANUNCIO, J. C. BASTOS, C. S. Seletividade fisiológica de inseticidas a Podisus nigrispinus (Dallas, 1851) (Heteroptera: Pentatomidae) predador de lagartas desfolhadoras de eucalipto. Revista Árvore, Viçosa, MG, v. 20, n. 3, p. 407-414, 1996.

YU, S. J. Selectivity of insecticides to the spined bug (Heteroptera: Pentatomidae) and its lepidopterous prey. Journal of Economic Entomology, Lanham, v. 81, n. 1, p. $119-122,1988$.

ZANUNCIO, J. C.; BATALHA, V. C.; GUEDES, R. N. C.; PICANÇO, M. C. Insecticide selectivity to Supputius cincticeps (Stal) (Het., Pentatomidae) and its prey Spodoptera frugiperda (J.E. Smith) (Lep., Noctuidae). Journal of Applied Entomology, Berlin, v. 122, n. 8, p. $457-460,1998$. 